\title{
Wiedemann-Steiner Syndrome as a Differential Diagnosis of Cornelia de Lange Syndrome Using Targeted Next-Generation Sequencing: A Case Report
}

\author{
Selma Demir ${ }^{\mathrm{a}}$ Hakan Gürkan ${ }^{\mathrm{a}}$ Veysel Öz $^{\mathrm{b}}$ Sinem Yalçıntepe ${ }^{\mathrm{a}}$ Emine İ. Atlı \\ Engin $A t l^{a}$
}

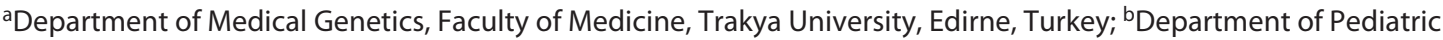
Neurology, Faculty of Medicine, Trakya University, Edirne, Turkey

\section{Established Facts}

- Wiedemann-Steiner syndrome is caused by pathogenic/likely pathogenic variations of the KMT2A gene which is one of the most frequently mutated genes implicated in intellectual disability. The associated phenotypes range from syndromic to nonsyndromic and from mild to severe intellectual disability.

- There are a number of previous reports indicating that Wiedemann-Steiner syndrome is one of the syndromes that can clinically and phenotypically overlap with Cornelia de Lange syndrome.

- Transcriptome disturbance caused by defects in the genes encoding the components of chromatin regulation and the transcription process is the common molecular mechanism of Cornelia de Lange and Wiedemann-Steiner syndromes.

\section{Novel Insights}

- The here reported likely pathogenic variation c.3647_3650delAAGA has been reported only once in a $\mathrm{PhD}$ thesis but not yet in the Leiden Open Variation Database nor in the literature.

- We believe that phenotypic information of the cases together with pathogenic/likely pathogenic variations is important, especially in rare diseases.

\section{Keywords}

de novo mutation $\cdot K M T 2 A \cdot$ Targeted next-generation

sequencing · Wiedemann-Steiner syndrome

\section{Abstract \\ Wiedemann-Steiner syndrome (WDSTS) is a rare autosomal dominant disorder with a variable clinical phenotype includ-}

ing synophrys, hypertelorism, thick eyebrows, long eyelashes, wide nasal bridge, long philtrum, hypertrichosis, growth retardation, and intellectual disability. Cornelia de Lange syndrome (CdLS) is a rare disease characterized by synophrys, long eyelashes, limb abnormalities, generalized hirsutism, growth retardation, and intellectual disability. In both WDSTS and CdLS, the malformations are due to transcriptome disturbance caused by defects in the genes encoding

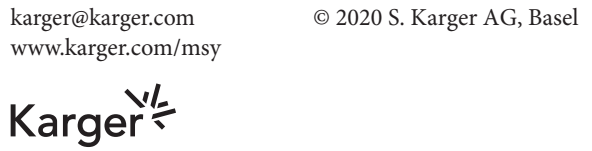

Selma Demir

Department of Medical Genetics, Faculty of Medicine

Trakya University, Balkan Campus, D300

Edirne 22030 (Turkey)

selmademir1980@gmail.com 
the components of chromatin regulation and transcription process. The overlapping features in these two syndromes may complicate the original diagnosis of a patient. Here, we report on a Wiedemann-Steiner patient found to have a de novo pathogenic KMT2A variation who had been clinically suspected as CdLS. We suggest that targeted next-generation sequencing is a feasible tool for the precise diagnosis of patients who have phenotypically and clinically overlapping features of CdLS and WDSTS.

(c) 2020 S. Karger AG, Basel

\section{Introduction}

Wiedemann-Steiner syndrome (WDSTS, OMIM \#605130), first described in 1989 [Wiedemann et al., 1989], is a rare autosomal dominant disorder with a variable clinical phenotype. Common facial features are hypertelorism, wide nasal bridge, long philtrum, thick eyebrows, synophrys, and long eyelashes. Patients generally have hypertrichosis cubiti or generalized hypertrichosis, developmental delay, mild to moderate intellectual disability, and behavioral abnormalities [Steiner and Marques, 2000; Koenig et al., 2010; Aggarwal et al., 2017]. Sacral dimple, rib abnormalities, and vertebral block have been reported in some patients [Baer et al., 2018]. The syndrome appears to be present among different populations and affects both genders equally. Although the syndrome is also known as one of the "hairy elbow syndromes," hypertrichosis cubiti may not be seen in all of the patients [Jones et al., 2012; Aggarwal et al., 2017; Baer et al., 2018; Li et al., 2018; Ramirez-Montaño and Pachajoa, 2019].

The genetic basis of WDSTS was first defined by Jones et al. [2012] after they found de novo pathogenic variations in the KMT2A gene in 5 out of 6 patients with distinctive facial appearance, hypertrichosis, and intellectual disability using whole-exome sequencing. In one out of the 6 patients they could not find a pathogenic variation by exome sequencing and suggested a distinct molecular genetic basis in this patient. KMT2A is a gene encoding a histone methyltransferase regulating chromatin-mediated transcription. Although the majority of the pathogenic variations of the KMT2A gene reported in WDSTS patients are premature termination mutations, missense mutations, splice site mutations, and exonic deletions in KMT2A have also been reported [Jones et al., 2012; Aggarwal et al., 2017; Lebrun et al., 2018].

Cornelia de Lange syndrome (CdLS) is a rare disease characterized by synophrys, long eyelashes, limb abnor-

Targeted Next-Generation Sequencing of Wiedemann-Steiner Syndrome

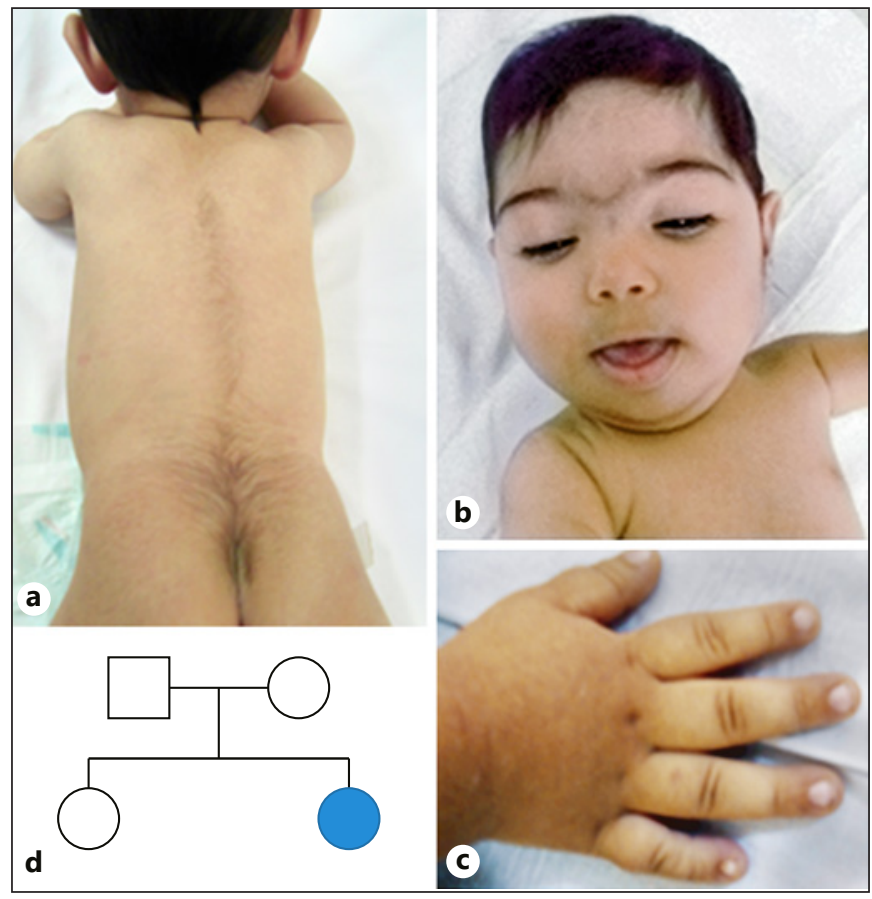

Fig. 1. a Hypertrichosis of the vertebral column, mostly in the lumbar and coccygeal region. b Distinctive facial features. c 5th finger clinodactyly. $\mathbf{d}$ Pedigree of the patient. The parents and the sister were sequenced and do not carry for the c.3647_3650delAAGA pathogenic variation in the KMT2A gene.

malities, generalized hirsutism, growth retardation, and intellectual disability. The syndrome has a wide range of some other manifestations like autistic and self-destructive behavior, cardiac septal defects, gastrointestinal dysfunction, hearing loss, myopia, and cryptorchidism or hypoplastic genitalia [Avagliano et al., 2020; Cascella and Muzio, 2020]. Pathogenic variations of NIPBL, SMC1A, $H D A C 8, S M C 3$, and RAD21 genes have been reported in patients with CdLS [Yuan et al., 2015; Aggarwal et al., 2017; Larizza and Finelli, 2019; Ramirez-Montaño and Pachajoa, 2019; Avagliano et al., 2020].

It may be hard to make a diagnosis of WDSTS because of the overlapping features of CdLS and WDSTS [Yuan et al., 2015; Jinxiu et al., 2020]. Both CdLS and WDSTS are known as disorders of transcriptional regulation. Global transcriptional disturbance due to mutations in the genes encoding components of chromatin regulation and transcription has been suggested to be responsible for the similar clinical and phenotypic features of these syndromes [Yuan et al., 2015].

Here, we report on a patient who had initially been suspected as CdLS based on the clinical examination but 


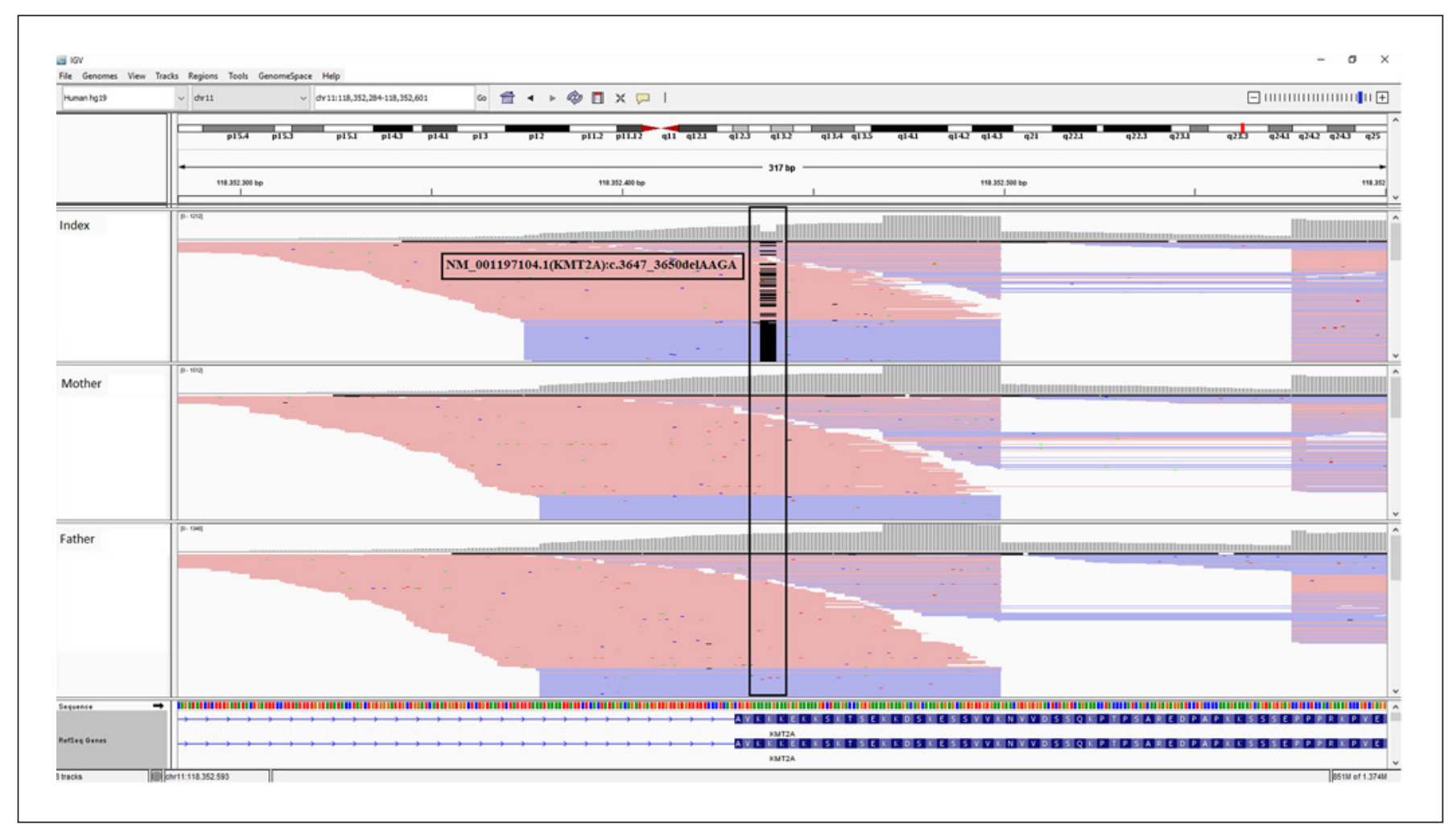

Fig. 2. IGV (https://software.broadinstitute.org/software/igv/) view of the NM_001197104.1(KMT2A):c.3647_ 3650delAAGA pathogenic variation in the index patient and her parents.

diagnosed as WDSTS after molecular genetic analysis. The c.3647_3650delAAGA (p.Lys1216ArgfsTer18) pathogenic variant in the KMT2A gene defined in our patient has been reported only once in a WDSTS patient in a PhD thesis [Jones, 2017] but not yet in the literature or in Leiden Open Variation Database. Also, here we want to emphasize the importance of targeted next-generation sequencing as a feasible tool for differentiating WDSTS and CdLS patients who have phenotypically and clinically overlapping features.

\section{Case Presentation}

An 8-month-old girl has been admitted to our genetic diagnosis center with suspicion of CdLS due to hypertrichosis, synophrys, dysmorphic features, and growth retardation. She was born as the second child of a nonconsanguineous couple at the 35th week of gestation via cesarean section. She had a weight of $3,050 \mathrm{~g}(10-25$ th centile), length of $48 \mathrm{~cm}$ (25th centile), and head circumference of $33 \mathrm{~cm}$ (10th centile) at birth. Because of respiratory distress, she had to stay in an incubator at the intensive care unit for 10 days after birth. When she was directed to our department, she was 8 months old. She was $65 \mathrm{~cm}$ tall (10-25th centile) with a weight of
$5,680 \mathrm{~g}(<3 \mathrm{rd}$ centile). Her head circumference was $40 \mathrm{~cm}(<3 \mathrm{rd}$ centile). She had an open fontanel and a narrow forehead. Physical examination revealed microcephaly, synophrys, ptosis, hypertelorism, thick eyebrows, long eyelashes, depressed and wide nasal bridge, long philtrum, thin upper lip vermilion, downturned corners of the mouth, high-arched palate, and micrognathia (Fig. 1). She had tapered fingers, brachydactyly, 5th finger clinodactyly in her right and a single transverse palmar crease in her left hand. Mild hypotonia was noted. She had hypertrichosis in the vertebral column, mostly in lumbar and coccygeal regions, but she did not have hypertrichosis cubiti. She started to control her head when she was 3 months old, but she was not able to sit unsupported when she was referred at the age of 8 months. The otolaryngologic and ophthalmologic examination revealed normal results except the bilateral ptosis.

Cytogenetic and molecular cytogenetic analysis results were normal. We did not define any pathogenic/likely pathogenic variation in coding regions of the NIPBL gene via Sanger sequencing (NM_133433.4) with in-house designed primers. We then performed next-generation sequencing with a targeted gene panel (QIAseq Targeted DNA Panel, Qiagen, Hilden, Germany) covering the coding regions of AFF4 (OMIM *604417), HDAC8 (*300269), KMT2A (*159555), NIPBL (*608667), RAD21 (*606462), SMC1A (*300040), SMC3 (*606062), and TAF6 $(* 602955)$ genes. Analysis of next-generation sequencing revealed a heterozygous NM_001197104.1(KMT2A):c.3647_ 
Table 1. Overlapping phenotypic and clinical features of disorders of transcriptional and chromatin regulation

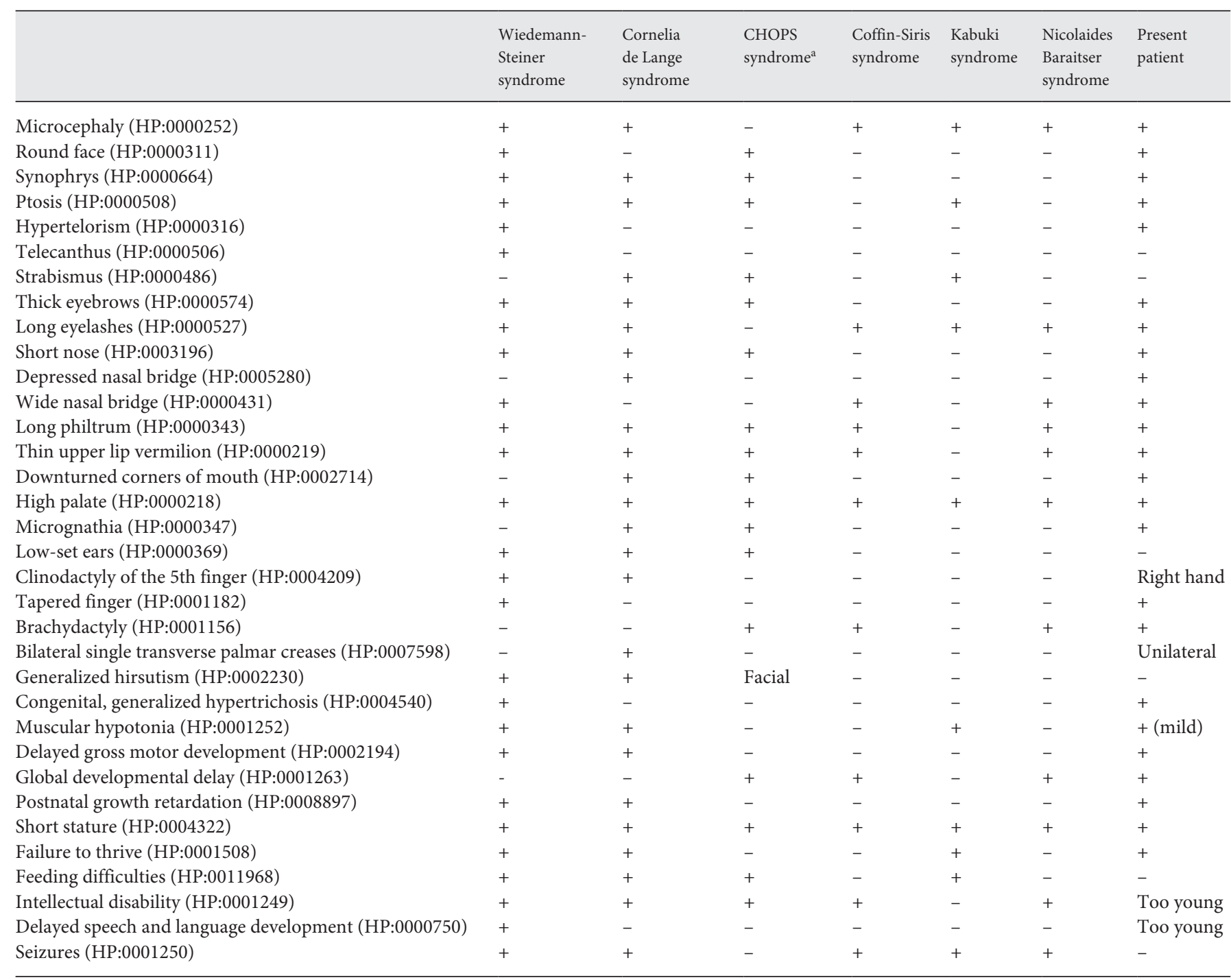

Adapted with the information included in https://www.orpha.net/consor/cgi-bin/Disease/Search.php?lng = EN. ${ }^{a}$ CHOPS, Cognitive impairment, coarse facies, heart defects, obesity, pulmonary involvement, short stature, and skeletal dysplasia.

3650delAAGA (p.Lys1216ArgfsTer18) variant (Fig. 2). This variant has been reported only once in a patient with WDSTS in a $\mathrm{PhD}$ thesis but not yet in Leiden Open Variation Database or in the literature [Jones, 2017]. This variant was not found in gnomAD [Karczewski et al., 2020]. Null variants in the KMT2A gene were reported to be pathogenic for WDSTS, where the loss of function is a known mechanism of disease [Lebrun et al., 2018]. Both parents and the clinically and phenotypically normal sister were not carrying the mutation. Altogether, these findings supported the pathogenicity of this de novo variation [Richards et al., 2015].

\section{Discussion and Conclusion}

In this study, we identified a de novo heterozygous pathogenic KMT2A variant in a patient with clinically and phenotypically overlapping features of CdLS and WDSTS. Molecular genetic analysis supported the diagnosis of WDSTS in the patient.

Syndromes associated with mutations in genes encoding components responsible for the transcriptional machinery and epigenetic modifications share some clinical manifestations including facial dysmorphism, growth problems, and intellectual disability [Izumi, 2016]. Transcriptome 
disturbance due to defects in genes encoding components of chromatin regulation and transcription mechanisms has been suggested to cause the similar clinical features of these syndromes [Yuan et al., 2015]. Cornelia de Lange, Wiedemann-Steiner, Coffin Siris, Kabuki, Nicolaides Baraitser, and CHOPS syndromes are from this group (Table 1) [Yuan et al., 2015; Izumi, 2016]. Based on the common clinical and phenotypic features, WDSTS and CdLS are the most similar syndromes of this group (Table 1). Although classical CdLS has some striking and recognizable clinical features, patients with milder phenotypes have been reported in the literature [Ireland and Burn, 1993; Izumi, 2016; Kaur et al., 2016]. Hypertrichosis cubiti has been reported as a distinctive feature of WDSTS. However, after the discovery of the causal gene, KMT2A, studies revealed patients with diffuse hypertrichosis without hypertrichosis cubiti [Jones et al., 2012; Aggarwal et al., 2017; Baer et al., 2018; Ramirez-Montaño and Pachajoa, 2019]. Hypertrichosis cubiti has been observed in just about $60 \%$ of patients in large case series whereas intellectual disability, long eyelashes, hypertrichosis of the back, wide nasal bridge, thick eyebrows, thin upper lip vermilion, and hypertelorism were the most frequent findings in the patients [Aggarwal et al., 2017; Baer et al., 2018; Li et al., 2018]. The phenotypic changes among patient populations with WDSTS may partly be attributable to different ages of the patients in the literature [Feldman et al., 2019; Ramirez-Montaño and Pachajoa, 2019]. Hypertrichosis in the present case was mainly detected in lumbar and coccygeal regions, but she did not have hypertrichosis cubiti. The presence of hypertrichosis in a number of syndromes, including CdLS, Coffin-Siris syndrome, and WDSTS, and other shared clinical features of these syndromes including cognitive delay, failure to thrive, and dysmorphic features challenge the distinction of these syndromes based on phenotypic information [Pavone et al., 2015; Pezzani et al., 2015].

Intellectual disability and developmental delay in mental/motor skills are common in Wiedemann-Steiner patients [Baer et al., 2018; Li et al., 2018]. The patient in this study was too young for assessment of intellectual disability, but she had mild delay in gross motor development compared to healthy peers. However, her motor developmental milestones were better than in WDSTS patients under 1 year in the literature [Chen et al., 2019; Arora et al., 2020].

The number of patients diagnosed with WDSTS increased dramatically after the discovery of KMT2A gene mutations as the underlying cause [Jones et al., 2012; Aggarwal et al., 2017; Baer et al., 2018; Li et al., 2018], and the number of studies reporting the diagnosis of WDSTS in patients whose tentative diagnoses were different is growing [Bramswig et al., 2015; Di Fede et al., 2020], emphasizing the importance of molecular genetic analyses as a key tool for clinical diagnosis. Bramswig et al. [2015] reported a patient with a KMT2A pathogenic variation defined by exome sequencing. The patient was a 22 -month-old boy with coarse face, hypertelorism, a broad nasal root and bulbous nasal tip, a full lower vermilion, long philtrum, upslanted palpebral fissures, long eyelashes, bilateral 5th finger clinodactyly and 5th fingernail hypoplasia, as well as hypertrichosis. His initial diagnosis was Coffin-Siris syndrome, which shares many phenotypic features with WDSTS [Bramswig et al., 2015]. Di Fede et al. [2020] reported 6 patients whose initial diagnosis was RubinsteinTaybi syndrome but who were found to have KMT2A pathogenic variations after sequencing analysis.

In this study, we used a targeted next-generation sequencing approach because the patient's clinical and phenotypic features were overlapping widely with CdLS and WDSTS, and we already have a designed targeted kit for molecular diagnosis of patients with overlapping features of CdLS, WDSTS, and CHOPS syndrome. In conclusion, we suggest that targeted next-generation sequencing is a valuable tool for clinicians to differentiate clinically and phenotypically overlapping syndromes like CdLS and WDSTS.

\section{Statement of Ethics}

The research was conducted ethically in accordance with the World Medical Association Declaration of Helsinki with the ethical approval of institutional board of Trakya University Faculty of Medicine. Written informed consent was obtained from the parents of the proband.

\section{Conflict of Interest Statement}

The authors have no conflicts of interest to declare.

\section{Funding Sources}

There is no funding of research relevant to this study.

\section{Author Contributions}

S.D. made substantial contributions to the conception of the work and wrote the manuscript. H.G. ensured that integrity of the whole work was appropriately investigated and resolved and approved the final manuscript. V.Ö. and S.Y. performed patient diagnosis and management and revised the intellectual content. E.I.A. and E.A. made substantial contributions to the analysis. 


\section{References}

Aggarwal A, Rodriguez-Buritica DF, Northrup H. Wiedemann-Steiner syndrome: Novel pathogenic variant and review of literature. Eur J Med Genet. 2017;60(6):285-8.

Arora V, Puri RD, Bijarnia-Mahay S, Verma IC. Expanding the phenotypic and genotypic spectrum of Wiedemann-Steiner syndrome: First patient from India. Am J Med Genet A. 2020;182(5):953-6.

Avagliano L, Parenti I, Grazioli P, Di Fede E, Parodi C, Mariani M, et al. Chromatinopathies: A focus on Cornelia de Lange syndrome. Clin Genet. 2020;97(1):3-11.

Baer S, Afenjar A, Smol T, Piton A, Gérard B, Alembik Y, et al. Wiedemann-Steiner syndrome as a major cause of syndromic intellectual disability: A study of 33 French cases. Clin Genet. 2018;94(1):141-52.

Bramswig NC, Lüdecke HJ, Alanay Y, Albrecht B, Barthelmie A, Boduroglu K, et al. Exome sequencing unravels unexpected differential diagnoses in individuals with the tentative diagnosis of Coffin-Siris and Nicolaides-Baraitser syndromes. Hum Genet. 2015;134(6):553-68.

Cascella M, Muzio MR. Cornelia de Lange Syndrome. In: StatPearls [Internet]. Treasure Island: StatPearls Publishing LLC; 2020.

Chen M, Liu R, Wu C, Li X, Wang Y. A novel de novo mutation (p.Pro1310Glnfs*46) in KMT2A caused Wiedemann-Steiner Syndrome in a Chinese boy with postnatal growth retardation: a case report. Mol Biol Rep. 2019; 46(5):5555-9.

Di Fede E, Massa V, Augello B, Squeo G, Scarano E, Perri AM, et al. Expanding the phenotype associated to KMT2A variants: overlapping clinical signs between Wiedemann-Steiner and Rubinstein-Taybi syndromes. Eur J Hum Genet. 2020.

Feldman HR, Dlouhy SR, Lah MD, Payne KK, Weaver DD. The progression of WiedemannSteiner syndrome in adulthood and two novel variants in the KMT2A gene. Am J Med Genet A. 2019;179(2):300-5.
Ireland M, Burn J. Cornelia de Lange syndrome-photo essay. Clin Dysmorphol. 1993;2:15160.

Izumi K. Disorders of Transcriptional Regulation: An Emerging Category of Multiple Malformation Syndromes. Mol Syndromol. 2016; 7(5):262-73.

Jinxiu L, Shuimei L, Ming X, Jonathan LC, Xiangju L, Wenyuan D. Wiedemann-Steiner syndrome with a de novo mutation in KMT2A: A case report. Medicine (Baltimore). 2020;99(16):e19813.

Jones WD. Genetic and Phenotypic Investigations into Developmental Disorders [dissertation]. Wellcome Trust Sanger Institute. Newnham College, University of Cambridge; 2017. https://www.sanger.ac.uk/research/ publications/theses.html

Jones WD, Dafou D, McEntagart M, Woollard WJ, Elmslie FV, Holder-Espinasse M, et al. De novo mutations in MLL cause WiedemannSteiner syndrome. Am J Hum Genet. 2012; 91(2):358-64.

Karczewski KJ, Francioli LC, Tiao G, Cummings BB, Alföldi J, Wang Q, et al. The mutational constraint spectrum quantified from variation in 141,456 humans. Nature. 2020; 581(7809):434-43.

Kaur M, Mehta D, Noon SE, Deardorff MA, Zhang Z, Krantz ID. NIPBL expression levels in CdLS probands as a predictor of mutation type and phenotypic severity. Am J Med Genet C Semin Med Genet. 2016;172(2):163-70.

Koenig R, Meinecke P, Kuechler A, Schäfer D, Müller D. Wiedemann-Steiner syndrome: three further cases. Am J Med Genet A. 2010; 152A(9):2372-5.

Larizza L, Finelli P. Developmental disorders with intellectual disability driven by chromatin dysregulation: Clinical overlaps and molecular mechanisms. Clin Genet. 2019;95(2):23140 .
Lebrun N, Giurgea I, Goldenberg A, Dieux A, Afenjar A, Ghoumid J, et al. Molecular and cellular issues of KMT2A variants involved in Wiedemann-Steiner syndrome. Eur J Hum Genet. 2018;26(1):107-16.

Li N, Wang Y, Yang Y, Wang P, Huang H, Xiong $\mathrm{S}$, et al. Description of the molecular and phenotypic spectrum of Wiedemann-Steiner syndrome in Chinese patients. Orphanet J Rare Dis. 2018;13(1):178.

Pavone P, Praticò AD, Falsaperla R, Ruggieri M, Zollino M, Corsello G, et al. Congenital generalized hypertrichosis: the skin as a clue to complex malformation syndromes. Ital J Pediatr. 2015;41:55.

Pezzani L, Milani D, Tadini G. Intellectual Disability: When the Hypertrichosis Is a Clue. J Pediatr Genet. 2015;4(3):154-8.

Ramirez-Montaño D, Pachajoa H. WiedemannSteiner syndrome with a novel pathogenic variant in KMT2A: a case report. Colomb Med (Cali). 2019;50:40-5.

Richards S, Aziz N, Bale S, Bick D, Das S, GastierFoster J, et al. Standards and guidelines for the interpretation of sequence variants: a joint consensus recommendation of the American College of Medical Genetics and Genomics and the Association for Molecular Pathology. Genet Med. 2015;17(5):405-24.

Steiner CE, Marques AP. Growth deficiency, mental retardation and unusual facies. Clin Dysmorphol. 2000;9(2):155-6.

Wiedemann HR, Kunze J, Grosse F-R, Dibbern H. A syndrome of abnormal facies, short stature, and psychomotor retardation. Atlas of Clinical Syndromes: A Visual Aid to Diagnosis for Clinicians and Practicing Physicians. London: Wolfe Publishing Ltd.; 1989. p. 198 9.

Yuan B, Pehlivan D, Karaca E, Patel N, Charng WL, Gambin T, et al. Global transcriptional disturbances underlie Cornelia de Lange syndrome and related phenotypes. J Clin Invest. 2015;125(2):636-51. 